\title{
Pé diabético e amputações em pessoas internadas em hospital público: estudo transversal
}

\author{
Diabetic foot and amputations on people in a public hospital: \\ cross-sectional study
}

Júlia de Cássia Oliveira ${ }^{1}$, Sara Alves dos Santos Taquary¹, Aurélio de Melo Barbosa², Rafaela Júlia Batista Veronezi,4

${ }^{1}$ Curso Multiprofissional em Clínica Especializada em Endocrinologia, Secretaria da Saúde do Estado de Goiás, Hospital Alberto Rassi (HGG) - Goiânia (GO), Brasil. ${ }^{2}$ Curso Multiprofissional em Clínica Especializada em Endocrinologia, Secretaria da Saúde do Estado de Goiás, Universidade Estadual de Goiás (UEG) - Goiânia (GO), Brasil.

${ }^{3}$ Secretaria da Saúde do Estado de Goiás - Goiânia (GO), Brasil.

${ }^{4}$ Faculdade Cambury - Goiânia (GO), Brasil.

\begin{abstract}
RESUMO
Introdução: O pé diabético é uma das mais devastadoras complicações crônicas do diabetes mellitus. Mais de $60 \%$ das amputações não traumáticas de membros inferiores ocorre em indivíduos diabéticos. Objetivo: Caracterizar sociodemográfica e clinicamente os pacientes hospitalizados por pé diabético, diferenciando o perfil entre amputados e não amputados. Métodos: Estudo transversal conduzido em hospital público no período de janeiro a junho de 2014. Foram incluídos indivíduos internados por pé diabético. Um formulário de dados foi utilizado e preenchido por meio de entrevista com pacientes e revisão de prontuários. Os dados sociodemográficos e clínicos foram analisados de acordo com a amostra total e os grupos de estudo: pacientes amputados e não amputados. Foi feita análise estatística descritiva e inferencial, sendo utilizado o teste de independência do $\chi^{2}$. Resultados: Quarenta e duas pessoas foram hospitalizadas por pé diabético, 26 sofreram amputação (61,9\%). Prevaleceu o sexo masculino entre os amputados e o feminino entre os não amputados. A faixa etária predominante foi a de 50 a 69 anos nos dois grupos. Houve associação estatística significativa entre as classificações de Wagner, PEDIS e a frequência de amputação, sendo que quanto maior o grau das classificações, maior a frequência de amputação. Conclusão: De maneira geral, os dados apresentados por este estudo foram semelhantes aos da literatura. As classificações de Wagner e PEDIS demonstraram-se úteis para identificar a gravidade do pé diabético.
\end{abstract}

Palavras-chave: pé diabético; amputação; estudos transversais.

\begin{abstract}
Introduction: The diabetic foot is one of the most devastating chronic complications of diabetes mellitus. More than $60 \%$ of non-traumatic lower limb amputations occur in diabetic people. Objective: To characterize sociodemographic and clinically the hospitalized patients for diabetic foot by differentiating the profile between amputees and not amputated subjects. Methods: A cross-sectional study was conducted in a public hospital from January to June 2014. We have included patients hospitalized due to diabetic foot. A data form was used and completed through interviews with patients and medical record review. The sociodemographic and clinical data were analyzed according to the total sample and study groups: the amputees and not amputated patients. Descriptive and inferential statistical analysis was done, using the $\chi^{2}$ test of independence. Results: Forty-two people were hospitalized due to diabetic foot and there were, 26 amputees (61.9\%). There was a prevalence of males among amputees and women among non-amputees. The predominant age was between 50 and 69 years in both groups. It was observed that there was a statistically significant association between classifications (Wagner and PEDIS) and frequency of amputation, and the higher the degree of classification, the higher the frequency of amputation. Conclusion: In general, data presented in this study are similar to the ones in literature. The classifications (Wagner and PEDIS) have shown to be useful to identify the severity of the diabetic foot.
\end{abstract}

Keywords: diabetic foot; amputation; cross-sectional studies.

Recebido em: 07/04/2015

Revisado em: 25/08/2015

Aprovado em: 24/11/2015

Autor para correspondência: Júlia de Cássia Oliveira - Hospital Alberto Rassi - Avenida Anhanguera, 6479 - Setor Oeste - CEP: $74110-010$ - Goiânia (GO),

Brasil - E-mail: julicassya@gmail.com

Conflito de interesses: nada a declarar. 


\section{INTRODUÇÃO}

Diabetes mellitus (DM) é um grupo de doenças metabólicas caracterizadas por hiperglicemia resultante de defeitos na secreção de insulina, na sua ação ou em ambos ${ }^{1}$. Na prática clínica, cerca de $90 \%$ dos casos são de DM tipo 2 e $10 \%$ são do tipo $1^{1}$. $\mathrm{O}$ DM tipo 2 permanece frequentemente não diagnosticado até que se manifestem sinais de complicações ${ }^{1}$, como nefropatia, retinopatia, neuropatia, acidente vascular cerebral, doença arterial coronariana e pé diabético ${ }^{2}$.

O pé diabético é uma das mais devastadoras complicações crônicas, atinge cerca de $15 \%$ dos pacientes com DM ao longo da vida. Caracteriza-se por infecção, ulceração ou destruição dos tecidos profundos associados a anormalidades neurológicas e a vários graus de doença vascular periférica nos membros inferiores. Apresenta grande repercussão social e econômica decorrente de amputações ${ }^{3}$. Mais de $60 \%$ das amputações não traumáticas de membros inferiores ocorrem em indivíduos diabéticos ${ }^{3}$, sendo que $85 \%$ dessas são precedidas por úlceras nos pés ${ }^{4}$.

A amputação associa-se ao aumento da mortalidade ${ }^{5}$ e é considerada maior quando realizada acima da articulação do tornozelo e menor quando realizada abaixo dessa ${ }^{6}$. O coto é denominado membro residual, sendo considerado um novo membro, responsável pelo controle da prótese durante a reabilitação ${ }^{7}$. Podem ocorrer complicações pós-operatórias no coto, tais como: deiscência de sutura, edema, ulceração e infecção ${ }^{8}$.

Este estudo teve como objetivo caracterizar sociodemográfica e clinicamente os pacientes hospitalizados por pé diabético em hospital público, diferenciando o perfil entre amputados e não amputados durante a hospitalização.

\section{MÉTODOS}

Estudo transversal conduzido em hospital geral, de ensino e pesquisa, especializado em média e alta complexidade, com foco eletivo, que oferta serviços terciários regulados pelo Sistema Único de Saúde (SUS), sendo unidade de referência para a região metropolitana de Goiânia e todo o estado de Goiás. A coleta de dados foi realizada no período de seis meses, de janeiro a junho de 2014, com busca ativa semanal, utilizando formulário de dados preenchido por duas fisioterapeutas residentes por meio de entrevista com pacientes e revisão de prontuários.

Foram incluídos pacientes internados por pé diabético que aceitaram participar da pesquisa por meio da assinatura do Termo de Consentimento Livre e Esclarecido, independente de sexo, idade, raça e procedência. As pessoas que foram internadas por outra causa e tiveram diagnóstico de pé diabético durante a internação não participaram.

As variáveis estudadas foram idade, sexo, índice de massa corporal (IMC), procedência, escolaridade, tipo de DM, tempo de diagnóstico, tipo de pé diabético, classificação de Wagner, classificação PEDIS (Perfusion, Extent, Depth, Infection, Sensation), presença de amputação prévia, complicações da internação, procedimentos cirúrgicos realizados, tempo de hospitalização e taxa de mortalidade.
O IMC foi mensurado utilizando a fórmula de Quetelet (IMC = peso corporal em quilogramas dividido pela altura em metros elevada ao quadrado $)^{4}$.

Segundo a classificação de Wagner ${ }^{5}$, o pé diabético pode ser classificado em:

- grau 0: risco elevado, ausência de úlcera;

- grau 1: úlcera superficial não infectada em termos clínicos;

- grau 2: úlcera profunda com ou sem celulite, ausência de abcesso ou osteomielite;

- grau 3: úlcera profunda com osteomielite ou formação de abcesso;

- grau 4: gangrena localizada;

- grau 5: gangrena em todo o pé.

A classificação PEDIS $^{9}$ possui os seguintes graus:

- grau I: ferida sem secreção purulenta, sem sinais inflamatórios;

- grau II: lesão que envolve apenas a pele ou o subcutâneo com a presença de mais dois sinais: calor local, eritema $>0,4$ a $2 \mathrm{~cm}$ ao redor da úlcera, dor local, edema local e drenagem de pus;

- grau III: eritema $>2 \mathrm{~cm}$, com um dos sinais citados ou que envolve estruturas mais profundas de infecção do que a pele e o subcutâneo - fasceíte, abcesso profundo, osteomielite e artrite;

- grau IV: qualquer infecção do pé na presença de síndrome da resposta inflamatória sistêmica.

Os dados foram analisados de acordo com a amostra total e os grupos de pacientes amputados e não amputados. Considerou-se amputado somente o indivíduo que passou pelo procedimento de amputação durante a internação pesquisada. $\mathrm{Na}$ análise estatística descritiva foi utilizado o programa Microsoft Excel® versão 2010. $\mathrm{Na}$ análise estatística inferencial foi utilizado o programa SPSS versão 15.0 , sendo realizado o teste de independência de $\chi^{2}$. $\mathrm{O}$ valor de significância adotado foi $\mathrm{p}<0,05$.

Este estudo ocorreu de acordo com os preceitos éticos determinados pela Resolução nº 466/2012 do Conselho Nacional de Saúde e outras regulamentações. Foi analisado pelo Comitê de Ética em Pesquisa do Hospital Alberto Rassi, com parecer consubstanciado de aprovação no 497.227, CAAE 21156913.7.0000.0035.

\section{RESULTADOS}

Do total de 42 indivíduos hospitalizados por pé diabético, 26 sofreram amputação (61,9\%). Prevaleceu o DM tipo 2 e somente um paciente do grupo dos não amputados apresentava o tipo 1. Os dados sociodemográficos e clínicos estão descritos na Tabela 1. Os sexos masculino e feminino tiveram o mesmo número de pacientes, porém prevaleceu o masculino entre os amputados e o feminino entre os não amputados, com diferença estatística significativa.

Quanto à escolaridade, grande parte das pessoas dos grupos tinha apenas o ensino fundamental incompleto. Novamente nos dois grupos, a faixa etária predominante foi 50 a 69 anos. Entre os 
amputados, a idade média foi 63,1 anos. A média da amostra geral foi próxima, 62,1 anos.

A procedência da região metropolitana de Goiânia foi a mais frequente no grupo dos não amputados, já a do interior de Goiás prevaleceu nos amputados, sem diferença estatística significativa. $\mathrm{O}$ peso normal e o sobrepeso foram os índices, classificados segundo o IMC, mais prevalentes em ambos os grupos. Porém, ao somar o percentual da amostra total de sobrepeso e obesidade, verifica-se que $54,8 \%$ dos indivíduos estavam acima do peso ideal.

Houve associação estatística significativa entre o tempo de diagnóstico e a amputação. Entre os amputados prevaleceu o tempo de diagnóstico até 10 anos e nos indivíduos não amputados prevaleceu o tempo superior a 10 anos. Três pessoas amputadas e um indivíduo não amputado, cerca de 9,5\% da amostra total, foram diagnosticados com DM apenas na hospitalização e desconheciam que sofriam essa enfermidade.

O tipo de pé diabético mais frequente na amostra geral e nos amputados foi o neuropático. No grupo dos não amputados foi maior o tipo isquêmico, sem diferença estatística significativa. A amputação prévia, realizada em internação anterior, ocorreu em pequena parcela do grupo de amputados, sendo maior nos não amputados.

Foram realizadas 27 amputações nos 26 pacientes que passaram por esse procedimento cirúrgico, sendo 15 maiores $(55,6 \%)$ e 12 menores $(44,4 \%)$. Outros procedimentos realizados: 8 debridamentos

Tabela 1: Características sociodemográficas e clínicas da amostra em estudo ( $n=42)$

\begin{tabular}{|c|c|c|c|c|c|}
\hline Variáveis & $\begin{array}{l}\text { Total } \\
\text { n (\%) }\end{array}$ & $\begin{array}{c}\text { Amputados } \\
\mathrm{n}(\%)\end{array}$ & $\begin{array}{c}\text { Não amputados } \\
n(\%)\end{array}$ & $\begin{array}{c}\text { Teste de } \\
\text { associação de } \chi^{2}\end{array}$ & $\begin{array}{l}\text { Associação } \\
\text { linear }\end{array}$ \\
\hline \multicolumn{6}{|l|}{ Sexo } \\
\hline Masculino & $21(50)$ & $17(65,4)$ & $4(25)$ & $\chi^{2}=6,46$ & \\
\hline \multirow[t]{2}{*}{ Feminino } & $21(50)$ & $9(34,6)$ & $12(75)$ & $p=0,011$ & \\
\hline & & & & $\varphi=0,39$ & \\
\hline \multicolumn{6}{|l|}{ Escolaridade } \\
\hline Sem instrução & $7(16,7)$ & $3(11,5)$ & $4(25)$ & $\chi^{2}=3,18$ & $z=0,0003$ \\
\hline Ensino fundamental incompleto & $25(59,5)$ & $17(65,4)$ & $8(50)$ & $p=0,36$ & $p=0,99$ \\
\hline Ensino fundamental completo & $5(11,9)$ & $4(15,4)$ & $1(6,3)$ & $V=0,27$ & \\
\hline Ensino médio completo & $5(11,9)$ & $2(7,7)$ & $3(18,8)$ & & \\
\hline \multicolumn{6}{|l|}{ Idade } \\
\hline $25-49$ anos & $3(7,1)$ & $1(3,8)$ & $2(12,5)$ & $\chi^{2}=1,71$ & $z=0,0007$ \\
\hline $50-69$ anos & $29(69)$ & $19(73,1)$ & $9(56,3)$ & $p=0,42$ & $p=0,97$ \\
\hline 70 anos ou mais & $10(23,8)$ & $6(23,1)$ & $5(31,3)$ & $V=0,20$ & \\
\hline \multicolumn{6}{|l|}{ Procedência } \\
\hline Região metropolitana & $21(50)$ & $10(38,5)$ & $11(68,8)$ & $\chi^{2}=3,88$ & $z=3,79$ \\
\hline Interior de Goiás & $20(47,6)$ & $15(57,7)$ & $5(31,3)$ & $p=0,14$ & $p=0,051$ \\
\hline Outros estados & $1(2,4)$ & $1(3,8)$ & $0(0)$ & $V=0,31$ & \\
\hline \multicolumn{6}{|l|}{ Índice de massa corporal } \\
\hline Baixo peso & $2(4,8)$ & $1(3,8)$ & $1(6,3)$ & $\chi^{2}=0,30$ & $z=0,01$ \\
\hline Peso normal & $17(40,5)$ & $11(42,3)$ & $6(37,5)$ & $\mathrm{p}=0,95$ & $p=0,91$ \\
\hline Sobrepeso & $17(40,5)$ & $10(38,5)$ & $7(43,8)$ & $\varphi=0,08$ & \\
\hline Obesidade & $6(14,3)$ & $4(15,4)$ & $2(12,5)$ & & \\
\hline \multicolumn{6}{|l|}{ Tempo de diagnóstico } \\
\hline Até 10 anos & $21(50)$ & $17(65,4)$ & $4(25)$ & $\chi^{2}=6,46$ & \\
\hline \multirow[t]{2}{*}{ Mais de 10 anos } & $21(50)$ & $9(34,6)$ & $12(75)$ & $p=0,011$ & \\
\hline & & & & $\varphi=0,39$ & \\
\hline \multicolumn{6}{|l|}{ Tipo de pé diabético } \\
\hline Neuropático & $16(38,1)$ & $11(42,3)$ & $5(31,3)$ & $\chi^{2}=1,98$ & $z=0,003$ \\
\hline Isquêmico & $13(31)$ & $6(23,1)$ & $7(43,8)$ & $p=0,37$ & $p=0,96$ \\
\hline Misto & $13(31)$ & $9(34,6)$ & $4(25)$ & $V=0,22$ & \\
\hline \multicolumn{6}{|l|}{ Amputação prévia } \\
\hline Não & $31(73,8)$ & $21(80,8)$ & $10(62,5)$ & $\chi^{2}=1,71$ & \\
\hline \multirow[t]{2}{*}{ Sim } & $11(26,2)$ & $5(19,2)$ & $6(37,5)$ & $p=0,19$ & \\
\hline & & & & $\varphi=0,20$ & \\
\hline
\end{tabular}


em 7 pacientes $(26,9 \%)$ e 4 cirurgias vasculares em 4 pacientes $(15,4 \%)$. Catorze pessoas do grupo de amputação (53,8\%) tiveram 32 complicações durante a internação, que consistiram em: 8 sepses (25\%), 6 pulmonares $(18,8 \%), 4$ renais $(12,5 \%), 4$ neurológicas, 3 gastrointestinais (9,4\%), 3 úlceras por pressão (9,4\%), 2 complicações cardíacas $(6,3 \%)$ e 2 infecções do coto (6,3\%). A taxa de mortalidade foi de $23,1 \%$ (6 óbitos), todos com quadro séptico.

Em relação aos 16 não amputados, foram realizados 5 debridamentos $(31,3 \%)$ e 5 cirurgias vasculares $(31,3 \%)$ em 5 pacientes diferentes. Houve 14 complicações em 9 pacientes, sendo: 6 complicações gastrointestinais $(42,9 \%), 4$ neurológicas (28,6\%), 2 renais (14,3\%), 1 pulmonar $(7,1 \%)$ e 1 sepse. Dois pacientes $(7,7 \%)$ foram a óbito devido à sepse ou complicação neurológica. A mortalidade na amostra geral foi de $19 \%$.

O tempo de hospitalização dos pacientes que amputaram variou de 7 a 157 dias, com média de 41,7 dias e desvio padrão de 35,8 dias. Foram empregados cuidados de Unidade de Terapia Intensiva (UTI) em 10 pessoas (38,5\%), com permanência média de 22,8 dias e desvio padrão de 47,3 dias, mínimo de 1 dia, máximo de 148 dias e mediana de 7 dias. O tempo de internação dos não amputados teve média e desvio padrão de 40,4 e 18,4 dias, respectivamente. Cinco pessoas (31,3\%) permaneceram na UTI com variação de 1 a 31 dias, média de 8 dias e desvio padrão de 12,9 dias.

Apresenta-se na Tabela 2 a associação entre a frequência de amputação e as classificações de Wagner e PEDIS por meio do teste do $\chi^{2}$. Nenhum paciente foi classificado como grau 0 na classificação de Wagner e grau I na PEDIS. Observa-se que houve associação estatística significativa com tendência linear, ou seja, quanto maior o grau nas classificações de Wagner e PEDIS, maior a frequência de amputação, com um pico no grau 4 da classificação de Wagner e grau III na PEDIS. Na amostra de amputados, prevaleceram os graus 3 e 4 na classificação de Wagner e III e IV na PEDIS. Foram mais frequentes a classificação de Wagner grau 4 e a PEDIS grau III na amostra geral.

\section{DISCUSSÃO}

Bortoletto et al.$^{10}$ afirmam que os homens são duas vezes mais submetidos à amputação do que as mulheres. $\mathrm{O}$ sexo masculino é um fator de risco para amputações em diabéticos tipo $2^{11}$. Os pacientes diabéticos amputados foram principalmente do sexo masculino. Resultado diferente do encontrado por Haddad, Bortoletto e Silva ${ }^{12}$, em que a maior parte dos internados amputados $(57,1 \%)$ constituiu-se de indivíduos do sexo feminino. Já outros estudos $^{13,14}$ também tiveram o sexo masculino predominando.

A média de idade observada na amostra geral (62,1 anos) foi próxima à de outros estudos, sendo 65; 62,7 e 59,4 anos ${ }^{4,15,16}$. A faixa etária acima de 60 anos tem abrangido a maior parte dos diabéticos amputados ${ }^{10,15,17}$. Isso é um fator que compromete ainda mais o processo de reabilitação, visto que o idoso desenvolve alteração na marcha e déficit de equilíbrio ao longo dos anos.

Semelhantemente a Haddad, Bortoletto e Silva ${ }^{12}$, a maior parte da amostra geral foi proveniente do município onde se localiza o hospital. Entretanto, a maioria dos amputados procedia do interior, assim como em uma pesquisa de Sergipe, onde $71 \%$ dos internados por pé diabético provinham do interior do estado ${ }^{6}$. Isso demonstra que o SUS tem referenciado pacientes de todo o estado de Goiás para o hospital público pesquisado, no qual há o acompanhamento de cirurgia vascular, endocrinologia, dentre outras especialidades médicas e multiprofissionais, como a psicologia e a fisioterapia.

O DM tipo 2 prevaleceu na amostra, tal como em outras pesquisas $^{12,16,18,19}$. O percentual de pacientes da amostra geral com diagnóstico no momento da internação $(9,5 \%)$ foi superior ao de um estudo $(4,8 \%)^{17}$ e inferior ao de outro $(17 \%)^{19}$. Na presente pesquisa, o tempo de diagnóstico até 10 anos foi predominante entre os amputados. Semelhantemente, o estudo de Santos et al. ${ }^{13}$ obteve o tempo de diagnóstico menor ou igual a 10 anos em maior número entre os amputados. Todavia, no estudo de Bortoletto et al. ${ }^{10}$, em mais da metade dos amputados ocorreu o tempo de diagnóstico maior do que 10 anos.

Obesidade ou sobrepeso estiveram presentes em mais da metade dos indivíduos estudados. Vale ressaltar que além de ser um fator

Tabela 2: Classificação do pé diabético da amostra em estudo $(n=42)$

\begin{tabular}{|l|c|c|c|c|c|}
\hline Variáveis & $\begin{array}{c}\text { Total } \\
\mathbf{n}(\%)\end{array}$ & $\begin{array}{c}\text { Amputados } \\
\mathbf{n}(\%)\end{array}$ & $\begin{array}{c}\text { Não amputados } \\
\mathbf{n}(\%)\end{array}$ & $\begin{array}{c}\text { Teste de } \\
\text { associação de } \chi^{2}\end{array}$ & $\begin{array}{c}\text { Associação } \\
\text { linear }\end{array}$ \\
\hline Classificação de Wagner & $4(9,5)$ & $0(0)$ & $4(25)$ & $\chi^{2}=14,25$ & $\mathrm{Z}=11,77$ \\
\hline 1 & $3(7,1)$ & $0(0)$ & $3(18,8)$ & $\mathrm{p}=0,007$ & $\mathrm{p}=0,0006$ \\
\hline 2 & $7(16,7)$ & $5(19,2)$ & $2(12,5)$ & $\mathrm{V}=0,58$ \\
\hline 3 & $26(61,9)$ & $19(73,1)$ & $7(43,8)$ & \\
\hline 5 & $2(4,8)$ & $2(7,7)$ & $0(0)$ & \\
\hline Classificação PEDIS & & & & \\
\hline II & $6(14,3)$ & $0(0)$ & $6(37,5)$ & $\chi^{2}=12,79$ \\
\hline III & $27(64,3)$ & $18(69,2)$ & $9(56,3)$ & $\mathrm{p}=0,002$ & $\mathrm{p}=0,001$ \\
\hline IV & $9(21,4)$ & $8(30,8)$ & $1(6,3)$ & $\mathrm{V}=0,55$ \\
\hline
\end{tabular}

PEDIS: perfusion, extent, depth, infection, sensation. 
complicador no controle do DM, a obesidade é considerada problema de saúde pública ${ }^{10}$.

Quanto à etiologia da ulceração do pé, tradicionalmente é citado que cerca de 45 a $60 \%$ das úlceras são puramente neuropáticas, cerca de $10 \%$ são puramente isquêmicas e que 25 a $45 \%$ são mistas (neuroisquêmicas) $^{5}$. Valores aproximados foram encontrados na amostra geral da pesquisa, em que $38,1 \%$ foi do tipo neuropático, $31 \%$ isquêmico e $31 \%$ do tipo misto. O estudo de Iribarren et al..$^{20}$ também apresentou valores próximos: 43; 36,4 e 20,6\%, respectivamente.

O relato de amputação prévia ocorreu em 19,2\% dos pacientes amputados, dado inferior ao observado em outras pesquisas, de 22 e $33,3 \%{ }^{6,12}$. Em relação à porcentagem de amputação realizada na internação, de $61,9 \%$, foi ligeiramente inferior ao valor encontrado por diferentes estudos, de 66,9 e $65 \%{ }^{19,20}$. Assim como observado na amostra da presente pesquisa (55,6\% de amputações maiores), um estudo ${ }^{15}$ verificou valor semelhante, $55,2 \%$ de amputações acima do tornozelo. Já em outras literaturas, as amputações menores prevaleceram ${ }^{17,21,22}$. Quanto a outros procedimentos realizados, o debridamento, realizado em $26,9 \%$ dos indivíduos amputados, também foi feito em $23,8 \%$ dos pacientes no Hospital Universitário de Londrina ${ }^{12}$.
O tempo de internação foi superior ao encontrado na literatura ${ }^{10,14,15}$. O maior tempo chegou a 157 dias, enquanto que em outros hospitais foram verificados o máximo de 50 e 117 dias ${ }^{10,14}$. A média de permanência na UTI $(22,8 \pm 47,3$ dias $)$ também foi muito superior à verificada por outra pesquisa ${ }^{10}$, de dois dias. A hospitalização prolongada ocorreu devido às complicações durante a internação, tais como sepse, insuficiência respiratória aguda e insuficiência renal aguda. O percentual de mortalidade no grupo de amputados $(23,1 \%)$ foi próximo ao relatado por outras pesquisas, de 18,5 e $28 \%{ }^{10,14}$. A taxa observada na amostra geral, de $19 \%$, também foi semelhante a outro estudo, de $17,3 \%^{20}$.

Assim como observado na amostra geral, diversos estudos ${ }^{6,15,16,19,23}$ verificaram prevalência do grau 4 da classificação de Wagner. Os graus 4 e 5 também estiveram associados frequentemente à ocorrência de amputação ${ }^{6,15}$.

De maneira geral, os dados apresentados por este estudo foram semelhantes aos encontrados na literatura. As classificações de Wagner e PEDIS demonstraram-se úteis para identificar a gravidade do pé diabético. Na prática clínica, especial atenção deve ser dada às pessoas do sexo masculino para que se evitem desfechos importantes, como a amputação.

\section{REFERÊNCIAS}

1. Przysiezny A, Rodrigues KF, Santiago LH, Silva MCV. Características sociodemográficas de pacientes com diabetes mellitus portadores de pé diabético e ou retinopatia diabética atendidos em 16 unidades de Estratégia de Saúde da Família de Blumenau. Arq Catarin Med. 2013;42(1):76-84

2. Morais GFC, Soares MJGO, Costa MML, Santos IBC. O diabético diante do tratamento, fatores de risco e complicações crônicas. Rev Enferm. 2009;17(2):240-5.

3. Almeida SA, Silveira MM, Santo PFE, Pereira RC, Salomé GM. Avaliação da qualidade de vida em pacientes com diabetes mellitus e pé ulcerado. Rev Bras Cir Plast. 2013;28(1):142-6. http://dx.doi.org/10.1590/S1983-51752013000100024

4. Santos ICRV, Sobreira CMM, Nunes ENS, Morais MCA. Prevalência e fatores associados a amputações por pé diabético. Cienc Saúde Coletiva. 2013;18(10):3007-14.

http://dx.doi.org/10.1590/S1413-81232013001000025

5. Duarte N, Gonçalves A. Pé diabético. Angiol Cir Vasc. 2011;7(2):65-79.

6. Nunes MAP, Resende KF, Castro AA, Pitta GBB, Figueiredo LFP, Miranda Junior F. Fatores predisponentes para amputação de membro inferior em pacientes diabéticos internados com pés ulcerados no estado de Sergipe. J Vasc Bras. 2006;5(2):123-30. http://dx.doi.org/10.1590/S1677-54492006000200008

7. Pastre CM, Salioni JF, Oliveira BAF, Micheletto M, Netto Junior J. Fisioterapia e amputação transtibial. Arq Ciênc Saúde. 2005;12(2):120-4.
8. Pires SR, Sandoval RA. Perfil de diabéticos amputados de membro inferior atendido no serviço de fisioterapia do Centro de Reabilitação e Readaptação Dr. Henrique Santillo CRER. Trances. 2010;2(4):213-24.

9. Santos ALG, Albino RB, Ortiz RT, Sakaki MH, Corsato MA, Fernandes TD. Neuroartropatia de Charcot: realinhamento do pé diabético por meio de osteossíntese com parafusos intramedulares: relato de caso. Rev Bras Ortop. 2014;49(5):535-9.

http://dx.doi.org/10.1016/j.rbo.2013.10.013

10. Bortoletto MSS, Viude DF, Haddad MCL, Karino ME. Caracterização dos portadores de diabetes submetidos à amputação de membros inferiores em Londrina, Estado do Paraná. Acta Sci Health Sci. 2010;32(2):205-13. http://dx.doi.org/10.4025/actascihealthsci.v32i2.7754

11. Assumpção EC, Pitta GB, Macedo ACL, Mendonça GB, Albuquerque LCA, Lyra LCB, et al. Comparação dos fatores de risco para amputações maiores e menores em pacientes diabéticos de um Programa de Saúde da Família. J Vasc Bras. 2009;8(2):133-8. http://dx.doi.org/10.1590/S1677-54492009000200006

12. Haddad MCL, Bortoletto MSS, Silva RS. Amputação de membros inferiores de portadores de diabetes mellitus: análise dos custos da internaçãoem hospital público. Cienc CuidSaúde. 2010;9(1):107-13. http://dx.doi.org/10.4025/cienccuidsaude.v9i1.10536

13. Santos ICRV, Nunes ENS, Melo CA, Farias DG. Amputações por pé diabético e fatores sociais: implicações para cuidados preventivos de enfermagem. Rev Rene. 2011;12(4):684-91. 
14. Tavares DMS, Dias FA, Araújo LR, Pereira GA. Perfil de clientes submetidos a amputações relacionadas ao diabetes mellitus. Rev Bras Enferm. 2009;62(6):825-30.

15. Rezende KF, Nunes MAP, Melo NH, Malerbi D, Chacra AR, Ferraz MB. Internações por pé diabético: comparação entre o custo direto estimado e o desembolso do SUS. Arq Bras Endrocrinol Metab. 2008;52(3):523-30

http://dx.doi.org/10.1590/S0004-27302008000300013

16. Torres-Aparcana HL, Gutiérrez C, Pajuelo-Ramírez J, PandoÁlvarez R, Arbañil-Huamán $H$. Características clínicas y epidemiológicas de los pacientes hospitalizados por pie diabético en el Hospital Nacional Dos de Mayo entre 2006 y 2008, Lima-Perú. Rev Peru Epidemiol. 2012;16(3):1-6.

17. Garcia YG, Pimentel DO, Valor IZ, Domínguez JAB, Pérez CV. Caracterización clínica de pacientes hospitalizados con una primera amputación de miembros inferiores por pie diabético. Rev Cuba Angiol Cir Vasc. 2012;13(2).

18. Herrera AG, Fernández RR, Ruiz VP, Hernández LR, Cabadilla LA, Sanabria RF, et al. El significado clínico del pie diabético en un análisis de diez años. Rev Cuba Angiol Cir Vasc. 2011;12(1).
19. Milman MHSA, Leme CBM, Borelli DT, Kater FR, Baccili ECDC, Rocha RCM, et al. Pé diabético: avaliação da evolução e custo hospitalar de pacientes internados no Conjunto Hospitalar de Sorocaba. Arq Bras Endocrinol Metab. 2001;45(5):447-51. http://dx.doi.org/10.1590/S0004-27302001000500007

20. Iribarren OB, Passim G, Aybar NM, Ríos P, González LA, Rojas MAG, et al. Pie diabético: evolución en una serie de 121 pacientes. Rev Chil Cir. 2007;59(5):337-41. http://dx.doi.org/10.4067/S0718-40262007000500005

21. Fernández FR, Martín TE, Alonso MER, Díaz IL. Frecuencia de amputaciones por pie diabético en un área de salud. Arch Med Camaguey. 2005;9(2).

22. Gurri DR. Caracterización de pacientes con pie diabético del Hospital Militar de Holguín. Correo Cient Med. 2012;16(1).

23. Vidal-Domínguez G, Mendoza-Cabrera $H$, Ticse-Aguirre RW Evaluación de la concordancia entre los valores del índice tobillo-brazo y presiones segmentarias con amputacion del pie diabético. Rev Soc Peru Med Interna. 2013;26(4)184-92. 